\title{
THE SIZE OF A GRAPH WITHOUT TOPOLOGICAL COMPLETE SUBGRAPHS*
}

\author{
M. CERA ${ }^{\dagger}$, A. DIÁNEZ ${ }^{\dagger}$, AND A. MÁRQUEZ
}

\begin{abstract}
In this note we show a new upperbound for the function $e x\left(n ; T K^{p}\right)$, i.e., the maximum number of edges of a graph of order $n$ not containing a subgraph homeomorphic to the complete graph of order $p$. Further, for $\left\lceil\frac{2 n+5}{3}\right\rceil \leq p<n$ we provide exact values for this function.
\end{abstract}

Key words. extremal graph theory, topological complete subgraphs

AMS subject classifications. 05C35, 05C70

PII. S0895480197315941

1. Introduction. As Bollobas says in [1], for many graph theorists one of the most general extremal problems is to find exact values of the function $e x\left(n ; T K^{p}\right)$, i.e., the maximum number of edges of a graph of order $n$ not containing a subgraph homeomorphic to $K^{p}$, where $K^{p}$ is the complete graph with $p$ vertices. But exact results for that function are only known for small values of $p$, trivially $e x\left(n, T K^{3}\right)=n-1(n \geq 3)$, and Dirac [2] proved that ex $\left(n, T K^{4}\right)=2 n-3(n \geq 3)$. For the case $p=5$, Dirac conjectured [3] that ex $\left(n, T K^{5}\right)=3 n-6$. But this value remains a conjecture and only some upperbounds due to Thomassen [7] are known, as $e x\left(n, T K^{5}\right) \leq 4 n-11$. For greater values of $p$, Mader [5] showed that for every $p$ there exists a constant $c_{p}$ such that $e x\left(n, T K^{p}\right) \leq c_{p} n(n \geq p)$. Later Mader [6] himself proved that

$$
e x\left(n ; T K^{p}\right) \leq t(p) n-\left(\begin{array}{c}
t(p)+1 \\
2
\end{array}\right)
$$

where $n \geq t(p)=3 \cdot 2^{p-3}-p$ with $p \geq 4$. And by considering the graph $K^{p-2}+\overline{K^{n-p+2}}$ it is easy to get that

$$
e x\left(n ; T K^{p}\right) \geq(p-2) n-\left(\begin{array}{c}
p-1 \\
2
\end{array}\right) .
$$

This inequality gives, actually, the exact results of the function for small values of $p$, but Mader [5] noticed, considering the complete 5-partite graph with two vertices in each class, that 7 is the greatest value of $p$ for which equality can hold.

The aim of this note is to provide an upperbound for the function $e x\left(n ; T K^{p}\right)$, showing that it is optimal in some situations, in such a way that for sufficiently large values of $n$ and $p$ the previous upperbound will be an equality. Actually, if $\left\lceil\frac{2 n+5}{3}\right\rceil \leq$ $p<n$, we will show exact values for $e x\left(n ; T K^{p}\right)$. Notations and terminologies not explicitly given here can be found in [1].

${ }^{*}$ Received by the editors January 31, 1997; accepted for publication (in revised form) February 1, 2000; published electronically May 15, 2000.

http://www.siam.org/journals/sidma/13-3/31594.html

${ }^{\dagger}$ E.T.S. Arquitectura, Universidad de Sevilla, Reina Mercedes 2, 41012-Sevilla, Spain (mcera@ cica.es, ana@apolo.us.es).

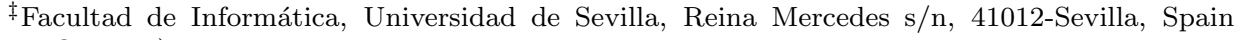
(almar@cica.es). 
2. Definitions and notations. The study of $e x\left(n, T K^{p}\right)$ may be stated equivalently as $\operatorname{ex}\left(n, T K^{n-q}\right)$ for appropriate values of $q$. In general, the technique we will apply to get our bounds is to choose $q$ vertices of a graph $G$ with $n$ vertices in such a way that the remaining $n-q$ vertices of $G$ will be the branch vertices of the subgraph of $G$ homeomorphic to $K^{n-q}$. (A vertex is called a branch vertex if its degree is not 2.)

In order to achieve the goal described above, we introduce some definitions and notations. For a graph $H$ and a set $\left\{v_{1}, \ldots, v_{q}\right\}$ of vertices of $H$, we denote by $H_{0}=H$ and by $H_{k}$ for $k=1, \ldots, q$ the induced subgraph in $H$ by the set of vertices $V(H)-\left\{v_{1}, \ldots, v_{k}\right\}\left(H_{k}=\left\langle V(H)-\left\{v_{1}, \ldots, v_{k}\right\}\right\rangle_{H}\right)$.

Most of the lemmas (as previous results) of this paper are devoted to obtaining graphs with certain prescribed properties, and those graphs will be used to prove the bounds given in the theorems. For the sake of simplicity (or in order to avoid excessive repetitions), we can say that if $r, t$ are two nonnegative integers, we define the family of graphs $\mathcal{C}_{r}^{t}$ as the set of graphs $H$ such that there exists a set $\left\{v_{1}, \ldots, v_{r}\right\}$ of vertices of $H$ verifying

(1) $\delta_{H_{j-1}}\left(v_{j}\right) \geq \delta_{H_{j}}\left(v_{j+1}\right)$, for $j=1, \ldots, r-1$.

(2) For each $h$ positive integer, if there exists $v \in H_{k}$ with $k=1, \ldots, r$ such that $\delta_{H_{k}}(v) \geq h$, then $\delta_{H_{j}}\left(v_{j+1}\right) \geq h$ for all $j=1, \ldots, k$.

(3) $H_{r}$ has at most $t$ edges $\left(\left|E\left(H_{r}\right)\right| \leq t\right)$.

First of all, we check that the families given above are nonempty.

LEMma 1. Let $H$ be a graph with $n$ vertices. Then, for any $r \leq n$, there exists $t$ such that $H$ is in $\mathcal{C}_{r}^{t}$.

Proof. Let $\delta_{0}$ be the maximum degree of $H$ and $\Delta_{0}$ the set of vertices of $H$ with degree $\delta_{0}$. Let $v_{1}$ be a vertex of $\Delta_{0}$. We consider $H_{1}=\left\langle V(H)-\left\{v_{1}\right\}\right\rangle_{H}$, $\delta_{1}=\max _{v \in V\left(H_{1}\right)}\left\{\delta_{H_{1}}(v)\right\}$, and $\Delta_{1}$ the set of vertices of $H_{1}$ with degree $\delta_{1}$. For each $v \in \Delta_{1}$ we note by $H_{1}(v)=\left\langle v_{1}, v\right\rangle_{H}$ and $\hat{\delta}_{1}=\max _{v \in \Delta_{1}}\left\{\delta_{H_{1}(v)}(v)\right\}$, we get $v_{2} \in \Delta_{1}$ such that $\delta_{H_{1}\left(v_{2}\right)}\left(v_{2}\right)=\hat{\delta}_{1}$. Then we may construct the chain of subgraph $\left\{H_{k}\right\}_{0 \leq k \leq r}$, noting by $\delta_{k}=\max _{v \in V\left(H_{k}\right)}\left\{\delta_{H_{k}}(v)\right\}$ and by $\Delta_{k}=\left\{v \in V\left(H_{k}\right) / \delta_{H_{k}}(v)=\delta_{k}\right\}$. For each $v \in \Delta_{k}$, let $H_{k}(v)$ be the subgraph $\left\langle v_{1}, \ldots, v_{k}, v\right\rangle_{H}$ and $\hat{\delta}_{k}=\max _{v \in \Delta_{k}}\left\{\delta_{H_{k}(v)}(v)\right\}$. We choose $v_{k+1} \in \Delta_{k}$ in such a way that $\delta_{H_{k}\left(v_{k+1}\right)}\left(v_{k+1}\right)=\hat{\delta_{k}}$. Hence, if $v \in$ $V\left(H_{k}\right)$ and $\delta_{H_{k}}(v) \geq h$, then $\delta_{H_{j}}\left(v_{j+1}\right) \geq h$ for $j \leq k$ with $k=1, \ldots, r$. The result follows.

In the previous lemma, the most important role is played by the vertices $(r)$, and we do not care about the number of edges $(t)$; obviously, from now on we will try to obtain tight values on the number of edges and from those values we will get our bounds.

3. An upperbound of $\operatorname{ex}\left(\mathbf{n}, \mathbf{T} K^{\mathbf{p}}\right)$. In this section, we provide a new upperbound of $\operatorname{ex}\left(n, T K^{p}\right)$; this upperbound will turn out to be the exact value of the function in many cases, as we will see in the next section.

First, we will give a sufficient condition on the edges of a graph to belong to the class $\mathcal{C}_{q}^{q}$.

Lemma 2. Let $n, q$ be two positive integers, with $q<n$. If $H$ is a graph with $n$ vertices and $2 q$ edges, then

(1) $H \in \mathcal{C}_{q}^{q}$.

(2) $\delta_{H_{q}}(v) \leq 1$ for $v \in V\left(H_{q}\right)$.

Proof. Let $H_{q}=\left\{v_{1}, \ldots, v_{q}\right\}$ be a set obtained as in Lemma 1. If there exists a vertex $v \in H_{q}$ such that $\delta_{H_{q}}(v) \geq 1$, then $\delta_{H_{j}}\left(v_{j+1}\right) \geq 1$ for $j=1, \ldots, q$. Thus 
$\left|E\left(H_{q}\right)\right| \leq q$, and therefore $H \in \mathcal{C}_{q}^{q}$. Furthermore, if there exists $v \in H_{q}$ such that $\delta_{H_{q}}(v) \geq 2$, then $\delta_{H_{j}}\left(v_{j+1}\right) \geq 2$ for every $j=1, \ldots, q$, and hence $|E(H)| \geq 2 q+2>q$, but this is not possible. Therefore, it follows that $\delta_{H_{q}}(v) \leq 1$ for $v \in V\left(H_{q}\right)$.

As we said before, once we have obtained in Lemma 2 an upperbound to the number of edges (refining, in this way, Lemma 1), we can get an upperbound to $e x\left(n, T K^{p}\right)$; thus, the first result related to this function is the following theorem.

Theorem 3. Let $n, p$ be two positive integers, with $n>p$. Then

$$
(p-2) n-\left(\begin{array}{c}
p-1 \\
2
\end{array}\right) \leq \operatorname{ex}\left(n, T K^{p}\right) \leq\left(\begin{array}{c}
n \\
2
\end{array}\right)-(2 n-2 p+1) .
$$

Proof. The upperbound is equivalent to

$$
e x\left(n, T K^{n-q}\right) \leq\left(\begin{array}{c}
n \\
2
\end{array}\right)-(2 q+1)
$$

where $q$ is a positive integer with $q<n$. Let $G$ be a graph with $\left(\begin{array}{c}n \\ 2\end{array}\right)-2 q$ edges. We will prove that $G$ contains a subgraph homeomorphic to $K^{n-q}$.

Let $H=\bar{G}$ be the complement of $G$. (The complement $\bar{G}$ of a graph $G$ also has $V(G)$ as its vertex set, but two vertices are adjacent in $\bar{G}$ if and only if they are not adjacent in $G$.) By Lemma 2, there exists a subset $\left\{v_{1}, \ldots, v_{q}\right\}$ of vertices of $G$ such that $s(s \leq q)$ nonadjacent edges $\left\{e_{1}, \ldots, e_{s}\right\}$ are missing in $G_{q}$ to create a complete graph of size $n-q$. We will show that the vertices of $G_{q}$ are going to be branch vertices of a subgraph of $G$ homeomorphic to $K^{n-q}$. Let $a_{i}, b_{i}$ be the vertices of the edge $e_{i}\left(e_{i}=\left(a_{i}, b_{i}\right)\right)$ for $i=1, \ldots, s$. There exists a path from $a_{i}$ to $b_{i}$ crossing a vertex of the set $\left\{v_{1}, \ldots, v_{q}\right\}$ for $i=1, \ldots, s$ and those paths are nonadjacent for each $i \in\{1, \ldots, s\}$. These assertions can be shown if we construct the following bipartite graph: the two nonadjacent vertices sets $X=\left\{e_{1}, \ldots, e_{s}\right\}$ and $Y=\left\{v_{1}, \ldots, v_{q}\right\}$, and a vertex $e_{i}$ of $X$, are joined with a vertex $v_{j}$ of $Y$ if there exists the path $a_{i} v_{j} b_{i}$ in $G$. This bipartite graph has a complete matching because if there exists $e_{i} \in X$ nonadjacent to $v_{q-i+1} \in Y$, then either $a_{i}$ or $b_{i}$ has degree at least 2 in $H_{q-i}$. And by Lemma 2 we know that $\delta_{H_{j-1}}\left(v_{j}\right) \geq 2$ for every $j=1, \ldots, q-i+1$, thus $|E(H)| \geq 2(q-i+1)+i-1+s>2 q$, but this is not possible. Hence, $G$ contains a subgraph homeomorphic to $K^{n-q}$.

4. Exact values for the function $\operatorname{ex}\left(\mathbf{n}, \mathbf{T K}^{\mathbf{P}}\right)$. The upperbound provided in Theorem 3 turns out to be the exact value of the function $\operatorname{ex}\left(n, T K^{p}\right)$, for a wide interval of $p$, as stated in the next theorem.

TheOREM 4. Let $n, p$ be two positive integers. If $\left\lceil\frac{3 n+2}{4}\right\rceil \leq p<n$, then

$$
e x\left(n, T K^{p}\right)=\left(\begin{array}{c}
n \\
2
\end{array}\right)-(2 n-2 p+1)
$$

Proof. Consider the graph obtained from $K^{n}$ removing $2 q+1$ nonadjacent edges (obviously, we need that $n \geq 4 q+2$ ). This graph does not contain a subgraph homeomorphic to $K^{n-q}$. Hence $e x\left(n ; T K^{n-q}\right)=\left(\begin{array}{c}n \\ 2\end{array}\right)-(2 q+1)$.

Now, let $G$ be a graph having $4 q-k+1$ vertices, with $q \geq 4$ and $0 \leq k \leq q-1$, in such a way that $\bar{G}$ is the graph formed by $k+1$ nonadjacent triangles and $2(q-k)-1$ nonadjacent edges, as in Figure 1. If we choose a set of $q$ vertices of $G$, it is evident that $\overline{G_{q}}$ has at least $q+1$ edges; hence the graph $G$ constructed does not contain a subgraph homeomorphic to $K^{n-q}$. 


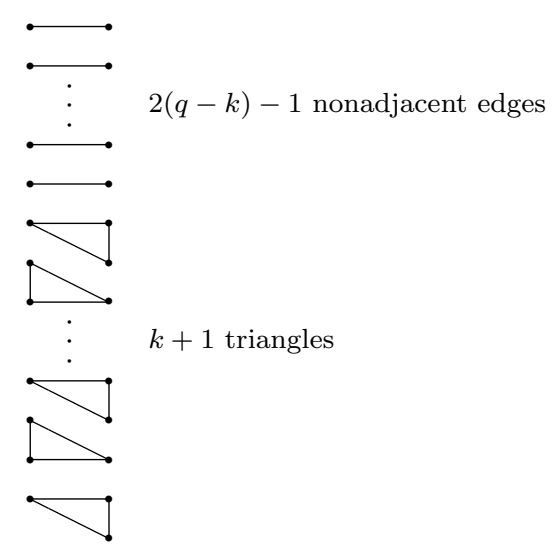

FIG. 1. Structure of $\bar{G}$.

Lemma 5. Let $q, k$ be two positive integers, with $q \geq 4$ and $0 \leq k \leq q-1$. It is verified that

$$
e x\left(4 q-k+1, T K^{3 q-k+1}\right) \geq\left(\begin{array}{c}
4 q-k+1 \\
2
\end{array}\right)-(2 q+k+2) .
$$

Our next aim is to prove that the previous inequality is, in fact, an equality. For that goal we need some prior results. First, we will show that given a graph with maximum degree 2 and at least $m$ vertices of maximum degree, it is possible to get at least $\left\lceil\frac{m+2}{3}\right\rceil$ nonadjacent vertices of maximal degree. We recall that given a graph $H$ and $v \in H$, the set of adjacent vertices to $v$ in $H$ is denoted by $\Gamma(v)$ (see [1]).

Lemma 6 . Let $k$ be a nonnegative integer and $H$ a graph with maximum degree 2 and at least $3 k+1$ vertices of maximum degree. Then there exist at least $k+1$ nonadjacent vertices with degree 2 .

Proof. We apply induction on $k$. For $k=1$ the result is obvious. Suppose now that $k>2$ and the result holds for $k-1$. Let $w$ be a vertex of $H$ of degree 2, we denote by $H^{*}=\langle\{v \in V(H)-\{\Gamma(w) \cup\{w\}\}\}\rangle_{H}$. Hence $H^{*}$ is a graph with at least $3 k+1-3=3(k-1)+1$ vertices with degree 2 and, by the induction hypothesis, $H^{*}$ has at least $(k-1)+1=k$ nonadjacent vertices $\left\{w_{1}, \ldots, w_{k}\right\}$ of degree 2 . Thus the $k+1$ vertices $w, w_{1}, \ldots, w_{k}$ are nonadjacent with degree 2 .

The next result basically asserts that given a positive integer $q$ and given $H$, a graph with maximum degree 2 whose number of vertices and edges depends on $q$, it is possible to get a set of vertices such that upon removing those vertices from $H$, the resulting graph has at most $q$ edges.

Lemma 7. Let $q, k$ be two nonnegative integers, $k \leq q-2$. Let $H$ be a graph with $4 q-k+1-i$ vertices and $2 q+k+1-2 i$ edges, $i \in\{1, \ldots, q\}$. If the maximum degree of $H$ is at most 2 , then $H \in \mathcal{C}_{q-i}^{q}$.

Proof. If $n_{j}$ denotes the number of vertices of degree $j$ in $H$, it is verified that

$$
\begin{aligned}
2 n_{2}+n_{1} & =2(2 q+k+1-2 i), \\
n_{2}+n_{1}+n_{0} & =4 q-k+1-i .
\end{aligned}
$$

From those equalities it is deduced that $n_{2}=3(k-i)+1+n_{0}$. For $i \leq k$, by Lemma 6 , the number of nonadjacent vertices with degree 2 is greater than or equal 
to $k-i+1$. We choose $\left\{v_{1}, \ldots, v_{q-i}\right\}$ as in Lemma 1 . So we guarantee that of the previous vertices at least $k-i+1$ have degree 2 , and, therefore, if we remove the vertices $\left\{v_{1}, \ldots, v_{q-i}\right\}$ from $H$, we would delete at least $2(k-i+1)+q-(k-i+1)$ edges. Thus we have that

$$
\left|E\left(H_{q-i}\right)\right| \leq 2 q+k+1-2 i-(2(k-i+1)+q-(k-i+1)) \leq q .
$$

For $i>k$, if we take the subset $\left\{v_{1}, \ldots, v_{q-i}\right\}$ of vertices of $H$ like in Lemma 1 , it is immediate that

$$
\left|E\left(H_{q-i}\right)\right| \leq 2 q+k+1-2 i-(q-i)=q+k-i+1 \leq q .
$$

Given a graph $H$ we will show that it is possible to obtain a set of $q$ vertices in such a way that if we remove them from $H$, then the graph $H_{q}$ has at most $q$ edges.

Lemma 8. Let $q, k$ be two positive integers with $k \leq q-2$. Let $H$ be a graph with $4 q-k+1$ vertices and $2 q+k+1$ edges. Then $H \in \mathcal{C}_{q}^{q}$.

Proof. First, suppose that the maximum degree of $H$ is 2 . In this case we have

$$
\begin{aligned}
2 n_{2}+n_{1} & =2(2 q+k+1), \\
n_{2}+n_{1}+n_{0} & =4 q-k+1 .
\end{aligned}
$$

From those equalities it is deduced that $n_{2}=3 k+1+n_{0}$ and, by Lemma 6 , the number of nonadjacent vertices with degree 2 is at least $k+1$. We take $\left\{v_{1}, \ldots, v_{q}\right\}$ as in Lemma 1. If the number of nonadjacent vertices of degree 2 is greater than or equal to $q$, it is obvious that $\left|E\left(H_{q}\right)\right| \leq q$. If, on the contrary, it is smaller than $q$, then if we remove the vertices $\left\{v_{1}, \ldots, v_{q}\right\}$ from $H$, we would be suppressing at least $2(k+1)+q-(k+1)$ edges and, therefore,

$$
\left|E\left(H_{q}\right)\right| \leq 2 q+k+1-(2(k+1)+q-(k+1))=q .
$$

If the maximum degree of $H$ is at least 3 , there exists $j \in\{1, \ldots, q\}$ such that the maximum degree of $H_{j}$ is smaller than or equal to 2 and upon applying Lemma 7 the result follows.

In order to show that we have an equality in Lemma 5 , the following theorem will be based on the same idea of the proof of Theorem 3. We start from a graph $G$ with a given number of vertices and edges and we will construct a bipartite graph in such a way that if we show the existence of a complete matching in this bipartite graph, then it will guarantee us that $G$ contains a subgraph homeomorphic to $K^{n-q}$. We recall Hall's condition for complete matching.

THEOREM 9 (see [4]). Given a bipartite graph with classes $X$ and $Y$, if $|\Gamma(A)| \geq|A|$ for all $A \subset X$, then there exists a complete matching, where $\Gamma(A)=\bigcup_{v \in A} \Gamma(v)$. Then

TheOrEm 10. Let $n, p$ be two positive integers with $\left\lceil\frac{2 n+5}{3}\right\rceil \leq p<\left\lceil\frac{3 n+2}{4}\right\rceil$.

$$
e x\left(n, T K^{p}\right)=\left(\begin{array}{c}
n \\
2
\end{array}\right)-(5 n-6 p+3) .
$$

Proof. It is equivalent to prove that

$$
e x\left(n ; T K^{n-q}\right)=\left(\begin{array}{c}
n \\
2
\end{array}\right)-(2 q+k+2)
$$


for $n=4 q-k+1$ with $q \geq 4,0 \leq k \leq q-4$.

Let $G$ be a graph with $\left(\begin{array}{c}n \\ 2\end{array}\right)-(2 q+k+1)$ edges. Let $\left\{v_{1}, \ldots, v_{q}\right\}$ be the set of vertices obtained in Lemma 8 by taking $H=\bar{G}$. Let $e_{1}=\left(a_{1}, b_{1}\right), \ldots, e_{s}=\left(a_{s}, b_{s}\right)$ be the edges of $H_{q}$. We will prove that there exists a path from $a_{i}$ to $b_{i}$ traversing through $\left\{v_{1}, \ldots, v_{q}\right\}$ for $i=1, \ldots, s$ and that those paths are nonadjacent for each $i \in\{1, \ldots, s\}$. For that, we consider the bipartite graph whose classes are $Y=\left\{v_{1}, \ldots, v_{q}\right\}$ and $X=\left\{e_{1}, \ldots, e_{s}\right\}$ such that $v_{j}$ is adjacent to $e_{i}$ in the bipartite graph if there exists the path $a_{i} v_{j} b_{i}$ in $G$. If we prove the existence of a complete matching in this bipartite graph we will have shown the result. Now we use Hall's condition to show the existence of complete matching. We get $i \in\{1, \ldots, q\}$. If $e_{i}$ is not adjacent to any vertex of the set $\left\{v_{q-2}, v_{q-1}, v_{q}\right\}$, then either $\delta_{H_{q}}\left(a_{i}\right) \geq 2$ or $\delta_{H_{q}}\left(b_{i}\right) \geq 2$ and, furthermore, either $\delta_{H_{q-2}}\left(a_{i}\right) \geq 3$ or $\delta_{H_{q-2}}\left(b_{i}\right) \geq 3$; hence $\delta_{H_{j-1}}\left(v_{j}\right) \geq 3$ for $j=1, \ldots, q-2$ and

$$
|E(H)| \geq 3(q-2)+4+s=3 q+s-2 .
$$

Since $k \leq q-3$ we would have that $|E(H)|>2 q+k+1$, but this is not possible, thus $\left|\Gamma\left(\left\{e_{i}\right\}\right)\right| \geq 1$.

We denote by $A_{i j}$ the set of vertices $\left\{e_{i}, e_{j}\right\}$ for $i, j \in\{1, \ldots, q\}$ with $i \neq j$. If $\Gamma\left(A_{i j}\right) \leq 1$, then at least three vertices of the set $\left\{v_{q-3}, v_{q-2}, v_{q-1}, v_{q}\right\}$ are adjacent neither to $e_{i}$ nor to $e_{j}$. Hence $\delta_{H_{j-1}}\left(v_{j}\right) \geq 3$ for $j=1, \ldots, q-3$ and

$$
\begin{aligned}
|E(H)| & \geq 3(q-3)+4+1+s=3 q+s-4 \\
& >2 q+k+1
\end{aligned}
$$

since $k \leq q-4$; therefore $\left|\Gamma\left(A_{i j}\right)\right| \geq 2$.

TABLE 1

Exact values of the function ex $\left(n, T K^{p}\right)$.

\begin{tabular}{|c|c|c|}
\hline$p$ & $e x\left(n, T K^{p}\right)$ & Reference \\
\hline \hline 3 & $n-1$ & {$[2]$} \\
\hline 4 & $3 n-6 ?($ conjecture $)$ & {$[3]$} \\
\hline$\vdots$ & $\vdots$ & $\vdots$ \\
\hline$\left\lceil\frac{2 n+5}{3}\right\rceil \leq p<\left\lceil\frac{3 n+2}{4}\right\rceil$ & $\left(\begin{array}{c}n \\
2\end{array}\right)-(5 n-6 p+3)$ & Theorem 10 \\
\hline$\left\lceil\frac{3 n+2}{4}\right\rceil \leq p<n$ & $\left(\begin{array}{c}n \\
2\end{array}\right)-(2 n-2 p+1)$ & Theorem 4 \\
\hline & & \\
\hline
\end{tabular}

For sets of vertices of size $m$ with $3 \leq m \leq s$ we consider $A_{i_{1}, \ldots, i_{m}}=\left\{e_{i_{1}}, \ldots, e_{i_{m}}\right\}$, where $\left\{i_{1}, \ldots, i_{m}\right\} \subset\{1, \ldots, q\}$ with $i_{1}<i_{2}<\cdots<i_{m}$. If $\Gamma\left(A_{i_{1}, \ldots, i_{m}}\right) \leq m-1$, then there exists at least one nonadjacent vertex to any element of $A_{i_{1}, \ldots, i_{m}}$ in the set of vertices $\left\{v_{q-(m-1)}, \ldots, v_{q}\right\}$; thus

$$
|E(H)| \geq(q-(m-1)) m+(m-1)+s,
$$


but for $3 \leq m \leq s, k \leq q-3$ we have that

$$
\begin{aligned}
(q-(m-1)) m+(m-1)+s & \geq m q-m^{2}+3 m-1 \\
& >2 q+k+1,
\end{aligned}
$$

and this is not possible. So $\Gamma\left(A_{i_{1}, \ldots, i_{m}}\right) \geq m$, and upon applying Hall's condition the result follows.

5. Conclusions. Up to now, exact values of the function $e x\left(n, T K^{p}\right)$ were known only for $p=3,4$. In this work, we provide a new upperbound to that function and we give its exact value when $\left\lceil\frac{2 n+5}{3}\right\rceil \leq p<n$. We can summarize our results in Table 1 .

\section{REFERENCES}

[1] B. Bollobas, Extremal Graph Theory, Academic Press, London, 1978.

[2] G. A. DiRAC, In abstrakten Graphen vorhandene vollständige 4-Graphenund ihre Unterteilungen, Math. Nachr., 22 (1960), pp. 61-85.

[3] G. A. Dirac, Homeomorphism theorem for graphs, Math. Ann., 153 (1964), pp. 69-80.

[4] P. Hall, On representatives of subsets, J. London Math. Soc., 10 (1935), pp. 26-30.

[5] W. MAder, Homomorphieegenshaften und mittlere Kantendichte von Graphen, Math. Ann., 174 (1967), pp. 265-268.

[6] W. MADER, Hinreichende Bedingungen für die Existenz von Teilgraphen, diezu einem vollständigen Graphen Homöomorph sind, Math. Nachr., 53 (1972), pp. 145-150.

[7] C. Thomassen, Some homomorphism properties of graphs, Math. Nachr., 64 (1974), pp. 119133. 